\title{
CMOS Optical Sensors for being incorporated in Endoscopic Capsule for Cancer Cells Detection
}

\author{
R. A. Dias, J. H. Correia, G. Minas \\ Dept. of Industrial Electronics, Laboratory of Biomedical Engineering \\ University of Minho \\ Campus de Gualtar, 4710-057 Braga, Portugal \\ Email: rosana@dei.uminho.pt, higino.correia@dei.uminho.pt, gminas@dei.uminho.pt
}

\begin{abstract}
This paper describes the design, fabrication and characterization of CMOS optical sensors for cancer detection based on autofluorescence emission spectra. These optical sensors were fabricated for being an integrated part of an endoscopic capsule used in diagnosis of gastrointestinal diseases. Cells and tissues show autofluorescence, in a part of the visible spectrum, when excited by ultraviolet or short-wavelength visible light. The emission spectrum of cancer tissue is different from the one of the healthy tissue, and, it is dependent on the cancer development stage. CMOS photodetectors can be used to measure that spectrum. Once they are small and easily integrated with readout electronics, they can be placed around all the surface of the endoscopic capsule. While, nowadays, a locomotion and stop mechanism is a challenge, this scheme can cover a large area of the gastrointestinal tract examination during the natural movement of the capsule. The complete system will innovate the way of physicians diagnose the gastrointestinal diseases.
\end{abstract}

\section{INTRODUCTION}

The most common methods for the gastrointestinal (GI) tract evaluation in medicine are endoscopic techniques. They allow visual access to different parts of the GI tract such as esophagus, stomach and intestine. However, some parts of the small intestine remain inaccessible (see Fig. 1) and a complete and efficient diagnosis can not be performed. This is the case of a patient that suffers from bleeding in the GI tract. Once the conventional endoscopy can not reach all the small intestine, a bleeding or suspicious point could not be found and, consequently, a diagnosis can not be performed. This situation is known as OGIB (Obscure Gastrointestinal Bleeding), i.e., the source of bleeding is not identified with upper endoscopy, colonoscopy and small intestine imaging study (five meters of small intestine can not be reachable) $[1,2]$.

To overcome those limitations it has appeared in the last years, the endoscopic capsule (EC). It is a relatively recent medical examination method whose practice is being increasing. In the future it intends to replace the endoscope (the tube placed inside the esophagus, stomach, intestine, etc.), which its medical examination is comfortless and requires experienced physicians.

The EC is a pill-sized wireless swallowable camera that travels (due to its swallowing) through the GI tract taking pictures. It comprises two batteries and an antenna through which it sends the obtained images to several sensors placed on the patient's abdomen [2]. The sensors are connected to a datarecorder that is fixed to a belt carried by the patient. At the end of the examination the data are downloaded to a workstation to produce a video. The EC image sensors are CCD or CMOS (Complementary Metal Oxide Semiconductor) imagers. The image is obtained with a $140^{\circ}$ field of view, 1:8 magnification, 1-30 $\mathrm{mm}$ depth of view and a minimum pixel-size of about $0.1 \mathrm{~mm}[1,2]$. The light source consists of two pairs of white LEDs (Light Emitting Diodes).

The capsule endoscopy has many advantages over the conventional endoscopy methods: it doesn't require the presence of an endoscopist during the procedure, it eliminates the need for sedation or insufflation and the patient can perform his normal daily activities. Above all, the capsule camera offers visual access, in a less invasive way, to parts of the small intestine that were previously accessed only by surgery. Because it is disposable, there's no risk of infection, but on the other hand, it adds more costs to the procedure: the capsule itself is expensive. As David Fleischer said in [1], "current capsule endoscopes are limited in that they cannot be maneuvered to specific sites; they cannot be returned to a site once it has been identified for further investigation; they do not allow for biopsy of tissue; and they cannot be used to deliver therapy".

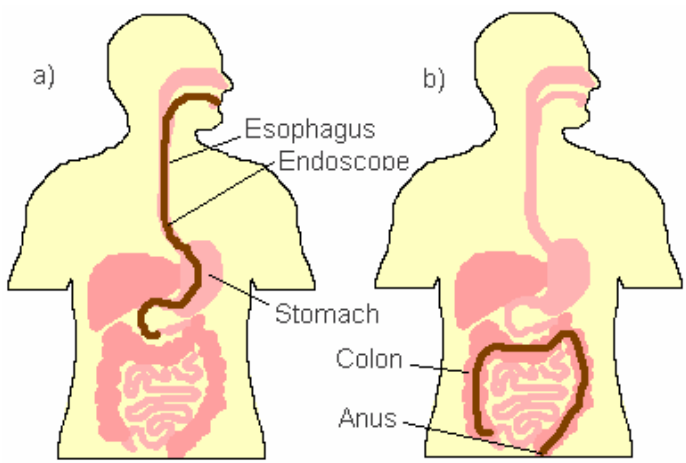

Fig. 1. a) Upper endoscopy and b) colonoscopy (lower endoscopy) procedure representation. 
Nowadays, early-stage cancer detection is a major goal in medicine, since it largely increases the survival rate. The EC may play an important role in this matter, once it can be equipped with miniaturized diagnosis and treatment instruments that can reach difficult areas. An EC equipped with these improvements may avoid the traditional surgical biopsy used for removing the tumor cells, which tissue needs to be sent to a laboratory for analysis, and the results become available after several hours, sometimes days.

Presently, some of the most promising methods for detecting cancer at an early stage are based on tissue's fluorescence. The abnormal tissue absorbs and emits light at specific wavelengths differently from the normal tissue, due to differences on endogenous chromophores and fluorophores concentrations and micro-distributions $[3,4]$.

This paper reports the design, fabrication and characterization of CMOS optical sensors capable of distinguish normal from cancerous tissue, in vivo, by measuring the tissue's autofluorescence spectrum when excited at a certain wavelength. These sensors could further be incorporated in the endoscopic capsule for detecting cancer cells during its traveling along the GI tract.

\section{CANCER CELLS AND DETECTION}

\section{A. Cells and Cancer}

The organs of the digestive system are covered by epithelial tissue which includes the squamous cells and the adenomatous cells. Once these cells are at tissue surface (in mucous membrane), they are suitable for analysis by sensors or even biosensors incorporated in the EC. These cells, when have mutations caused by chemical agents, physical agents, genetic mutation, among others, are transformed themselves in carcinomas (a malignant tumor of epithelial origin) and adenocarcinoma (a malignant tumor of glandular epithelium), respectively. Squamous cell carcinoma and adenocarcinoma are the most common kind of esophagus cancer being the adenocarcinoma the most common kind of stomach and small intestine cancer $[5,6]$. The spectral response of adenomatous cells (Fig. 2) in epithelial tissue in presence and absence of adenocarcinoma is studied for proper design of CMOS optical sensors.

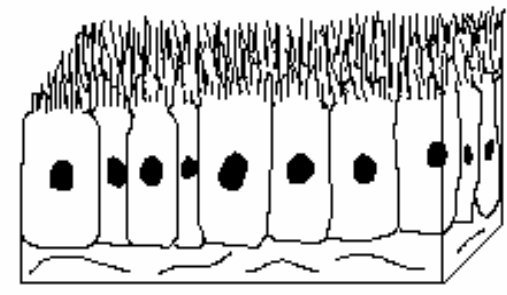

Fig. 2. Adenomatous cells representation.

\section{B. Autofluorescence Emission Principle}

Epithelial tissue presents autofluorescence when excited by ultraviolet or short-wavelength visible light $(400-550 \mathrm{~nm})$. In this light condition, certain biomolecules present mainly in the submucosa of the epithelium, such as the endogenous fluorophores, emanate light at longer wavelengths [3]. Examples are the green-fluorescing collagen and elastin molecules, NADH, flavin, tryptophan and porphyrin $[3,7,8]$. The emission wavelength range depends on the different groups of fluorophores that were activated. Each group has different excitation wavelengths. The emission of porphyrin is in the red spectral range, while the others are in the blue and green ranges [9].

In the ultraviolet and visible part of the spectrum, the optical properties of the tissue are dominated by the endogenous chromophores (light-absorbing biomolecules) which are different for normal and abnormal tissue and have different fluorescent spectra as well [4].

Since neoplastic tissue (tissue with formation of tumors) has higher blood irrigation and is usually thicker than normal tissue, less light is transmitted through the mucosa to the submucosa. Thus, less light excites the endogenous fluorophores present in the submucosa. As a consequence, less fluorescent light (emitted by those fluorophores) is transmitted through the neoplastic tissue for detection, once hemoglobin absorbs most of the light [9]. Therefore, the autofluorescence level of an epithelial tissue with adenocarcinoma is lower than normal tissue and it can be used to distinguish the presence and absence of an adenocarcinoma (see Fig. 3).

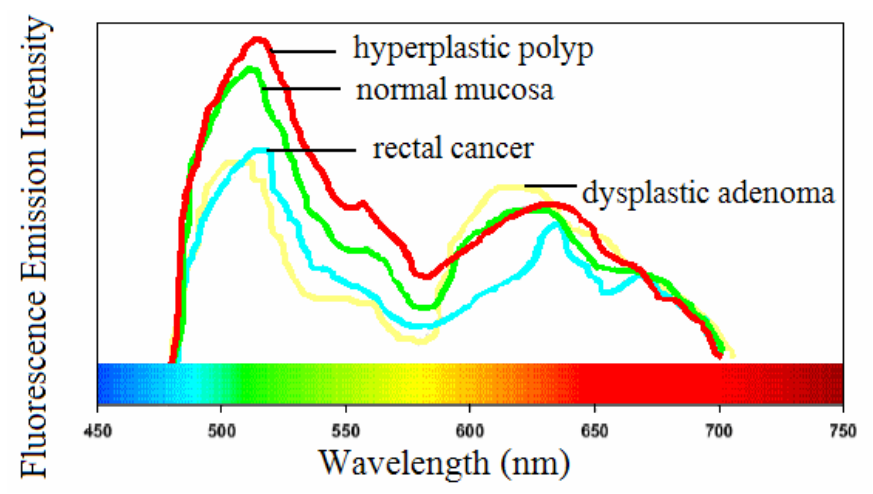

Fig. 3. Fluorescence behavior representation (mean spectra) of some colorectal tissues.

Several in vivo samples of healthy mucosa were studied in order to find spectral patterns similarities between all those autofluorescence spectra. The same study has been performed for adenocarcinoma [9-11]. The obtained shape of the healthy mucosa and the adenocarcinoma spectra is similar (Fig. 3). They have two characteristic peaks, one in the green range ( $\lambda$ around $515 \mathrm{~nm}$ ) and the other in the red range ( $\lambda$ around 
$640 \mathrm{~nm}$ ). However, the green peak (around 510-515 nm) shows different intensities depending on the cancer stage. The authors concluded that the better wavelength for differentiation between the fluorescent spectrum of a healthy mucosa and an adenocarcinoma is around $510-515 \mathrm{~nm}$. Therefore, the CMOS optical sensors should be developed for having the higher quantum efficiency in that spectral range.

\section{CMOS OPTICAL SENSOR DESIGN}

The optical sensors should be incorporated in an EC for measuring the spectral autofluorescence of epithelial tissue. Moreover, they should have integrated in the same die their readout circuits for converting the analog photodetectors signal into a digital signal, with a suitable format for RF transmission.

The economic viability of that detection system critically depends on the possibility of realizing the system in a standard microelectronic process (preferable CMOS) Furthermore, the system would have to fit in an unaltered CMOS process, using the layers available only and without additional process steps. Therefore, the CMOS optical sensors design involves selection of the photodetector type available in a CMOS standard process. Fortunately, in the visible part of the spectrum, the absorption of light in silicon is wavelength dependent and the response can be programmed by design. A deeper junction shows in general an enhanced long wavelength response. Selecting the suitable junction depth allows to set a maximum peak at a specific wavelength [12]. In addition, the spectral response is determined by the thickness and material properties of the dielectric layers available in the CMOS standard process. The arrangement of those different layers forms an interference filter with the interference peak at different wavelengths.

The photodetectors are pn-junction photodiodes, fabricated using the $n+/ p$-epilayer junction (Fig. 4). This structure is chosen because it provides the best possible quantum efficiency in the desired spectral range, due to the different doping concentration between the $\mathrm{n}$ and the $\mathrm{p}$ side, which extends the $\mathrm{p}$ side depletion area more deeply [13].

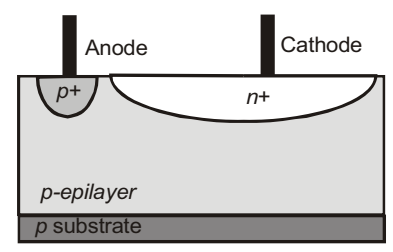

Fig. 4. Cross-section of the fabricated pn-junction photodiode.

An additional photodiode for measuring the photodiode dark current is introduced in the circuit. The dark current is the current that flows in a photodiode when there is no optical radiation incident on it. It is usually measured and subtracted from the flux. Since the dark current is temperature dependent, one measurement at the beginning of the experiment is usually not sufficient. Thus, in the reported circuit, a dark current compensation channel is implemented.

It is desirable to integrate the analog-to-digital conversion and the photodetectors in the same die. A CMOS sigma-delta converter is designed. It produces a bit stream signal and allows its use in small data-acquisition and control systems. The oversampling principle allows a flexible trade-off between bandwidth and accuracy (noise). Since the bandwidth of the CMOS circuits is much higher than the required bandwidth for the light measurements, a simple first-order sigma-delta converter with a high oversampling ratio can be used. The sigma-delta output signal is proportional to the photodiode current and hence proportional to the fluorescent light emitted by the tissue. The converter should be designed with a small number of transistors, occupying a very small area in the device, which enables the introduction of several photodetectors.

\section{CMOS OptiCAL SENSOR FABRiCATION}

The CMOS compatible photodetectors and readout circuits have been fabricated through a double-metal, singlepolysilicon, $0.7 \mu \mathrm{m}$ n-well CMOS process. Since technology rules from the CMOS process have to be met, the design of the optical path is restricted to combinations of the three dielectric layers available on top the pn-junction. An oxide layer, with a thickness around $600 \mathrm{~nm}$, can increase the transmittance from $50 \%$ to $60 \%$, but also introduces wavelength dependence (see Fig. 5). To eliminate the wavelength dependence of the transmission through this layer, in some cases, the best choice would be the removal of all dielectric layers from the top of the silicon surface. This process is performed at the design level, using the same masks designed for the metal contacts and for the overlayer (without additional masks or steps). However, in the used standard CMOS process, due to fabrications constraints, only the overlayer can be removed. The first oxide layer (a thin oxide) and the second oxide must remain (Fig. 6).

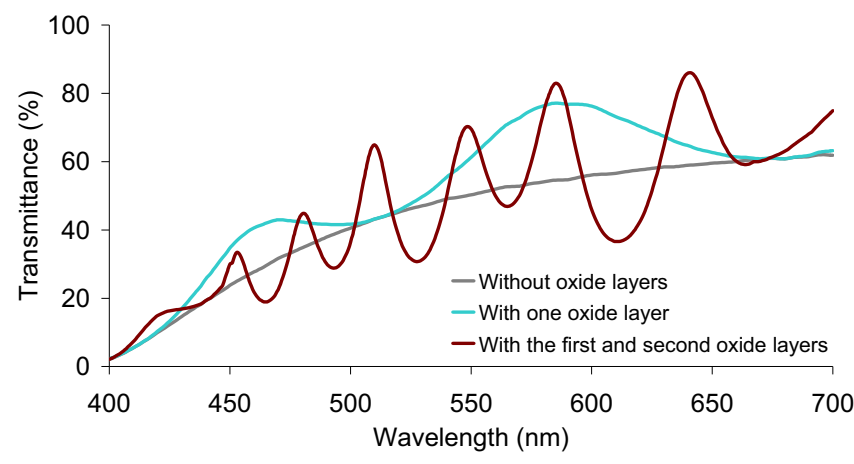

Fig. 5. Simulated spectral responses of some combinations of the dielectric layers above the photodiode pn-junction. The simulations are done with an optics software package TFCalc 3.4, supplied by Software Spectra, Inc., USA. 


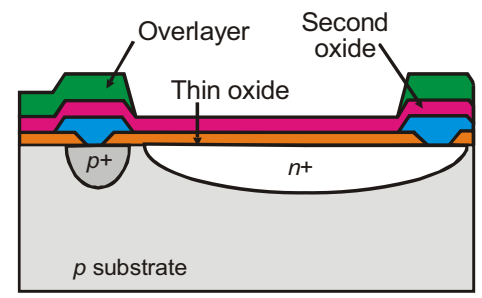

Fig. 6. Cross-section of the fabricated CMOS photodiode structure (including the dielectric layers above the pn-junction).

There have been fabricated three different sizes of photodetectors. Fig. 7 shows a photograph of the fabricated photodiodes. The photodiodes D1 and D2 have an active are of $100 \times 100 \mu \mathrm{m}$. They have two oxide layers and D2 has also the overlayer. D3 has an active area of $50 \times 50 \mu \mathrm{m}$ and D4 of $25 \times 25 \mu \mathrm{m}$. The photodiode for measuring the dark current is point out with the letter ' $\mathrm{B}$ '. A metal layer covers its active area.

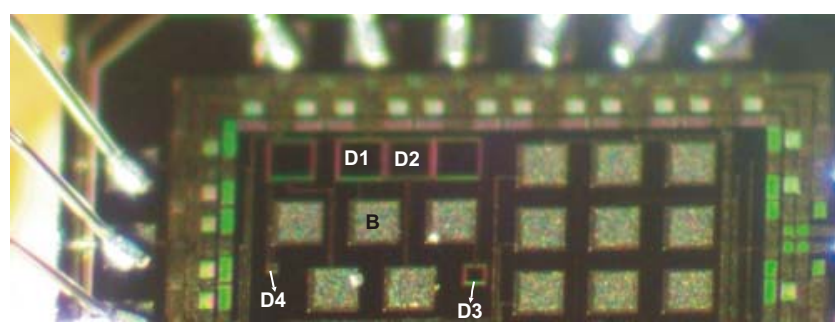

Fig. 7. A photograph of the CMOS photodetectors.

\section{EXPERIMENTAL RESULTS}

The electrical characteristics and the spectral responsivity of the photodiodes were measured using a Keithley 487 picoamperemeter and a $250 \mathrm{~W}$ quartz tungsten halogen lamp with the ORIEL Cornerstone $130^{\mathrm{TM}}$ monochromator used as light source. The on-chip photodiodes were calibrated with a calibrated commercially available photodiode as reference (Hamamatsu S1336-5BQ).

The photodiodes dark current for several reverse bias voltage are shown in Fig. 8. A dark current of $0.64 \mathrm{pA}, 0.52 \mathrm{pA}$, $0.35 \mathrm{pA}$ and $0.27 \mathrm{pA}$ at $0 \mathrm{~V}$ was obtained for the photodiodes D2, D1, D3 and D4, respectively.

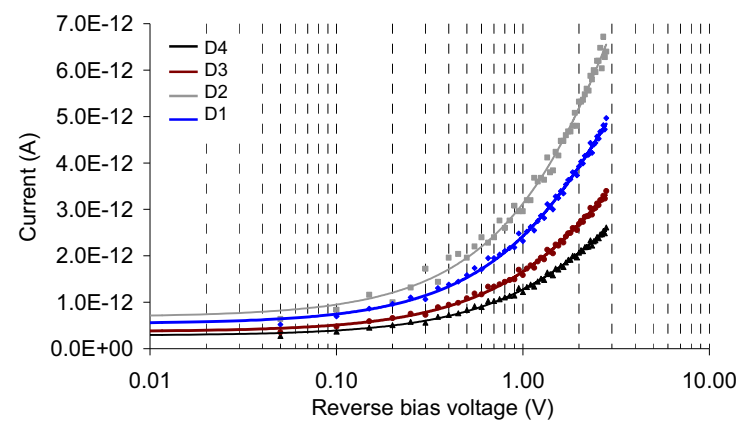

Fig. 8. D1, D2, D3 and D4 dark current versus reverse bias voltage.
The spectral response and the quantum efficiency curves of the fabricated photodiodes, measured using the calibrated Hamamatsu photodiode as reference, are shown in Fig. 9 and Fig. 10, respectively. Equation (1) was used for determining the quantum efficiency from the responsivity values,

$$
\Re=\frac{I_{P}}{P_{i}}=\frac{q \eta \lambda}{h c}
$$

where $\mathfrak{R}$ is the responsivity, $I_{p}$ the measured photodiode current, $P_{i}$ the incident optical power, $q$ the electron charge, $\eta$ the quantum efficiency, $\lambda$ the wavelength, $h$ the Plank constant and $c$ the light velocity.

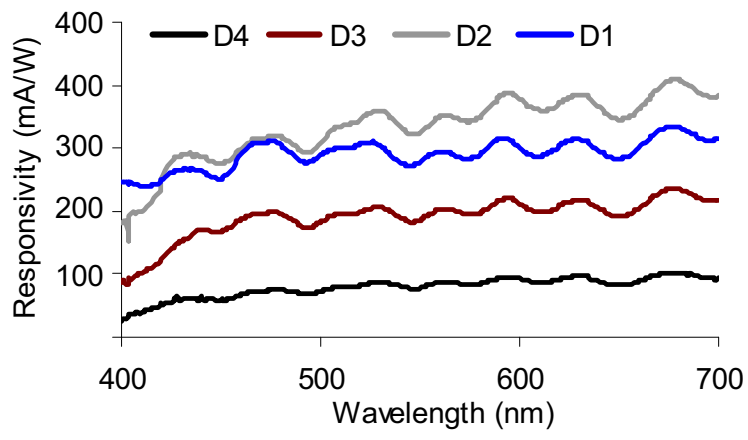

Fig. 9. Measured responsivity using the calibrated Hamamatsu commercial photodiode as a reference.

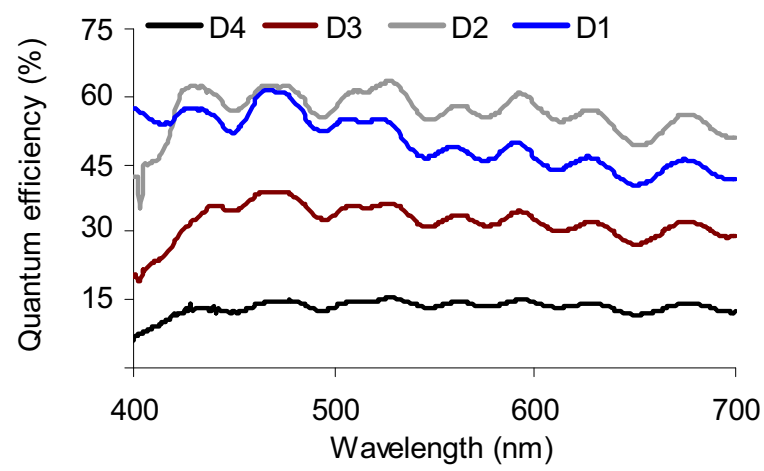

Fig. 10. Measured quantum efficiency calculated using equation (1).

From Fig. 9 and Fig. 10 it can be seen the effect of the overlayer in D2. D1 showed to be the best featured photodiode in almost the complete bandwidth. Layout optimizations for superior quantum efficiency of the small photodiodes are on going, especially if it will be necessary to miniaturize the system and use a large detection area. However, for now, D1 can be used for the characterization of cancer cells by fluorescence.

\section{CONCLUSIONS}

The on-chip integrated CMOS optical sensors presented here have been developed for being integrated in an endoscopic 
capsule for measuring the emission spectrum of normal and cancer cells. The emission spectrum of cancer and healthy tissue has different intensities and it is dependent on the cancer development stage. The optical sensors are CMOS photodetectors optimized for the wavelength range where the spectrum of cancer and healthy cells have the higher deviation. However, the autofluorescence level of cancer tissue can be, in certain cases, similar to the one of a vessel or inflammation. To overcome this and for not confusing inflammation with the begin state of an adenocarcinoma, a reflectance measurement within $650 \mathrm{~nm}$ to $700 \mathrm{~nm}$ might be necessary. The CMOS photodiodes suitable for that measurement are on going using a deeper junction (n-well/p-epilayer).

This scheme of measuring the spectral emission avoids the need to produce a video of the traveling area of the gastrointestinal tract. However, these photodiodes can also be used in an array to form a CMOS image matrix sensor.

\section{ACKNOWLEDGMENT}

The authors wish to acknowledge Paulo Mendes, from Dept. of Industrial Electronics, University of Minho, for his help with the wire bonding of the device.

\section{REFERENCES}

[1] D. Fleisher, "Capsule imaging", Clinical Gastrology and Hepatology, vol. 3, pp. s30-s32, July 2005.
[2] M. Delvaux and G. Gay "Capsule endoscopy in 2005: Facts and Perspectives," Best Practice \& Research Clinical Gastroenterology, vol. 20, pp. 23-39, 2006.

[3] R.S. DaCosta, B.C. Wilson, and N.E. Marcon, "Spectroscopy and fluorescence in esophageal diseases," Best Practice \& Research Clinical Gastroenterology, vol. 20, pp. 41-57, February 2006.

[4] M. Stringer and K. Moghissi, "Photodiagnosis and fluorescence imaging in clinical practice," Photodiagnosis and Photodynamic Therapy, vol. 1, pp.9-12, May 2004.

[5] C.S. Huang, C.C. Luo, J.N. Lin, and S.F. Huang, "Adenocarcinoma of the jejunum: a pediatric case report," Chang Gung Med. J., vol. 26, pp. 204-207, March 2003

[6] T. Takahashi, N. Iwama, "Atypical glands in gastric adenoma," in Virchows Archiv, vol. 403, Springer Berlin, Heidelberg, pp. 135-148, June 1984.

[7] E. Endlicher and H. Messmann, "Spectroscopy and fluorescence imaging," Techniques in Gastrointestinal Endoscopy, vol. 5, pp.74-77, April 2003.

[8] S.L. Jacques, "Tissue fluorescence," Proc. Soc. Photo. Opt. Instr. Eng., vol. 2371, pp. 2-13, 1995.

[9] B. Mayinger, P. Horner, M. Jordan, C. Gerlach, T. Horbach, W. Hohenberger, and E.G. Hahn, "Light-induced autofluorescence spectroscopy for tissue diagnosis of GI lesions," Gastrointestinal Endoscopy, vol. 52, pp. 395-400, September 2000.

[10] B. Mayinger, M. Jordan, T. Horbach, P. Horner, C. Gerlach, S. Mueller, W. Hohenberger, and E.G. Hahn, "Evaluation of in vivo endoscopic autofluorescence spectroscopy in gastric cancer," Gastrointestinal Endoscopy, vol. 59, pp. 191-198, February 2004.

[11] B. Mayinger, M. Jordan, P. Horner, C. Gerlach, S. Muehldorfer, B.R. Bittorf, et al., "Endoscopic light-induced autofluorescence spectroscopy for the diagnosis of colorectal cancer and adenoma," Journal of Photochemistry and Photobiology B: Biology, vol. 70, pp. 13-20, April 2003.

[12] H. R. Philipp and E. A. Taft: Optical constants of silicon in the region 1 to 10 ev. Physical Review, 120/1 (1960), 37-38.

[13] G. Minas, J.C. Ribeiro, R.F. Wolffenbuttel and J.H. Correia, "On-Chip integrated CMOS optical detection microsystem for spectrophotometric analyses in biological microfluidic systems," Proceedings of ISIE 2005, pp. 1133-1138, June 2005 